\title{
The Impact of Insecticide Pre-Exposure on Longevity, Feeding Succession, and Egg Batch Size of Wild Anopheles gambiae s.l.
}

\author{
Grace Msangi, ${ }^{1}$ Moses I. Olotu, ${ }^{1}$ Aneth M. Mahande, ${ }^{2}$ Anitha Philbert, ${ }^{3}$ \\ and Eliningaya J. Kweka $\mathbb{D}^{4,5}$ \\ ${ }^{1}$ Department of Biological Sciences, Mkwawa University College of Education, P.O. Box 2513, Iringa, Tanzania \\ ${ }^{2}$ Division of Livestock and Human Health Disease Vector Control, Tropical Pesticides Research Institute, Mabogini Field Station, \\ Moshi, Tanzania \\ ${ }^{3}$ Department of Zoology and Wildife Conservation, University of Dar Es Salaam, Dar Es Salaam, Tanzania \\ ${ }^{4}$ Tropical Pesticides Research Institute, Division of Livestock and Human Diseases Vector Control, Mosquito Section, \\ P.O. Box 3024, Arusha, Tanzania \\ ${ }^{5}$ Department of Medical Parasitology and Entomology, Catholic University of Health and Allied Sciences, P.O. Box 1464, \\ Mwanza, Tanzania
}

Correspondence should be addressed to Eliningaya J. Kweka; pat.kweka@gmail.com

Received 16 April 2020; Revised 11 September 2020; Accepted 19 September 2020; Published 28 September 2020

Academic Editor: Aditya Prasad Dash

Copyright (C) 2020 Grace Msangi et al. This is an open access article distributed under the Creative Commons Attribution License, which permits unrestricted use, distribution, and reproduction in any medium, provided the original work is properly cited.

\begin{abstract}
Background. Insecticide resistance among the vector population is the main threat to existing control tools available. The current vector control management options rely on applications of recommended public health insecticides, mainly pyrethroids through long-lasting insecticidal nets (LLINs) and indoor residual spraying (IRS). Regular monitoring of insecticide resistance does not provide information on important factors that affect parasite transmission. Such factors include vector longevity, vector competence, feeding success, and fecundity. This study investigated the impacts of insecticide resistance on longevity, feeding behaviour, and egg batch size of Anopheles gambiae s.l. Method. The larval sampling was conducted in rice fields using a standard dipper $(350 \mathrm{ml})$ and reared to adults in field insectary. A WHO susceptibility test was conducted using standard treated permethrin $(0.75 \%)$ and deltamethrin $(0.05 \%)$ papers. The susceptible Kisumu strain was used for reference. Feeding succession and egg batch size were monitored for all survivors and control. Results. The results revealed that mortality rates declined by 52.5 and $59.5 \%$ for permethrin and deltamethrin, respectively. The mortality rate for the Kisumu susceptible strain was 100\%. The survival rates of wild An. gambiae s.l. was between 24 and 27 days. However, the Kisumu susceptible strain blood meal feeding was significantly higher than resistant colony $(t=2.789, \mathrm{df}=21, P=0.011)$. Additionally, the susceptible An. gambiae s.s. laid more eggs than the resistant An.gambiae s.l. colony $\left(\mathrm{X}^{2}=1366, \mathrm{df}=1, P \leq 0.05\right)$. Conclusion. It can, therefore, be concluded that the wild An. gambiae s.l. had increased longevity, blood feeding, and small egg batch size compared to Kisumu susceptible colonies.
\end{abstract}

\section{Introduction}

Malaria is still one of the most prevalent human vectorborne disease that threatens the world's population living in areas where there is a risk of infectious bites with 228 million morbidity and 405000 mortality cases reported in 2018 [1]. About $90 \%$ of all malaria deaths in the world occur in subSaharan Africa (SSA) due to a combination of factors such as availability of predominant malaria vectors and parasites, as well as local conducive weather conditions [2-7]. Children under the age of five and pregnant women are the most vulnerable groups [1]. Globally, there are 490 species of Anopheles, of which only 70 are considered as potential malaria vectors [8]. Currently, species such as Anopheles gambiae s.s., An. arabiensis, An. merus, An. melus, An. coluzzii, and $A n$. funestus members are the main malaria vectors that are responsible for spread of the disease in SSA [9-15]. So far, vector control measures remain a main intervention strategy for the global malaria eradication programme [16-19]. The core vector control measures that are 
widely used include long-lasting insecticidal nets (LLINs) and indoor residual spraying (IRS) [1]. The strategy aimed to reduce the risk of malaria infection by targeting indoor biting mosquitoes $[1,20]$. Despite the effectiveness of these two interventions in malaria control, they are not sufficient to control residual malaria [21-23]. However, the main challenges associated with LLINs and IRS are insecticide resistance, improper use of the interventions, host behaviour, such as staying outdoor during early night or sleeping outdoor without using protective measures, and vector behaviour including outdoor biting and outdoor resting [21].

Insecticide resistance has now been reported in malaria vector against the four classes of public health insecticides used in malaria vector control, and it was estimated that, in 2016, resistance would have been reported in 71 malaria endemic countries [24] and may continue to threaten sustainability of malaria interventions. So far, pyrethroids have been the only class used for LLINs and contributed for a large proportion of the insecticide used for IRS. Insecticide resistance occurs due to the use of the same chemical insecticides of public health repeatedly $[25,26]$ or multiple exposures of vectors to different sources of insecticides. It has also been associated with higher doses application than the ones recommended for malaria control [25, 27]. Different resistance mechanisms recorded so far are metabolic resistance, target-site mechanism, and behavioural resistance, as well as cuticle-reduced penetration resistance $[25,27,28]$. In the target-site mechanism, the configuration that occurs in the amino acid leads to a less active or inactive binding site [27-29]. Similarly, malaria vectors also have the ability to produce high amount of enzyme naturally, which enable them to metabolize the insecticide and become functionless [30]. Other insects modify their cuticle or the digestive track by slowing down or prevent the absorption of the insecticides penetrations [31], while behavioural resistance occurs after a long time exposure of insects to an insecticide $[24,27,32,33]$. The resistance mechanism has been confirmed to help mosquitoes in enduring endless insecticide stress, and when these mechanism actions are high, physiological characteristics such as mosquito longevity, larva development, reproduction, or its blood feeding ability may be affected [34-36]. For example, permethrin has been reported to reduce the blood feeding ability of $A n$. arabiensis [37], An. stephensi, and Ae. aegypti [36]. It significantly reduced the egg batch size up to $100 \%$ to the resistant colony of Ae. aegypti [38]. The resistance status with multiple blood meals increases the survival of the vectors and tolerance of the resistance with aging [39], while in a normal scenario without multiple feeding, the resistance decreases with aging [32]. Occurrence of insecticide resistance among malaria vectors is an impediment to the disease control efforts. In response to this problem, the World Health Organization has put in place the information systems for insecticide resistance monitoring [40]. Nevertheless, most other important factors that determine the ability of the vector to transmit the parasites are underexplored during the day-to-day insecticide resistance monitoring activities. Such factors include vector longevity, biting behavior, and vector competence of the insecticide-resistant malaria vectors, and their impact on malaria epidemiology is underexplored [41, 42]. To be successfully transmitted, Plasmodium parasites must complete their life cycle from gametocytes in the blood meal to sporozoites in the saliva [41]. This means that vectors should live long enough to allow such development of the parasite. Previous studies reported that insecticide resistance genes in vector mosquitoes can cause pleiotropic effects to other genes and, hence, may affect the resistant vectors either negatively or positively by interfering with their fecundity, longevity, and or vector competence $[42,43]$. In addition, the insecticide susceptibility test demonstrated mortality beyond the 24 hours of the WHO susceptibility test in resistant mosquitoes, implying the shortening of the parasite transmission potentials [44]. However, studies to explore the interplay between vector resistance with longevity and fecundity are scanty. Most studies have been conducted in a laboratory setting using laboratory-reared colonies. It was important to explore these factors from the natural environment. The present study investigated the impact of insecticide preexposure on longevity, feeding behaviour, and egg batch size of An. gambiae s.l. from northern Tanzania.

\section{Materials and Methods}

2.1. Study Site. This study was conducted at Mabogini Village in Moshi District, Kilimanjaro Region, northeastern Tanzania. The experimental site was located between latitude $03^{\circ} 21^{\prime} \mathrm{S}$ and longitude $37^{\circ} 20^{\prime}$ E $750 \mathrm{~m}$ above the sea level, covering an area of 1600 ha [45]. The site is well known for anthropogenic activities, mainly rice irrigation that contributes to significant breeding sites of mosquitoes [46]. The annual temperature ranges from $18.0^{\circ} \mathrm{C}$ to $30.7^{\circ} \mathrm{C}$, while the average annual rainfall is $525 \mathrm{~mm}$ between March and May with a shorter period of rainfall occurring between November and January [47].

2.2. Larval Sampling. Mosquito larvae and pupae were collected from rice paddy fields by using a standard dipper (350 $\mathrm{ml}$ ladle) and reared in plastic containers [48]. All sampled larvae were morphologically identified and pooled into groups according to their species using morphological keys [49]. The sampled larvae were reared at the Mabogini field station, Moshi, close to rice irrigation schemes.

2.3. Rearing of Mosquito Larvae. All collected Anopheles gambiae s.l were reared in plastic containers of $18 \times 18 \times 18 \mathrm{~cm}$ with fresh water. Larvae were reared under insectary conditions, temperature $27^{\circ} \mathrm{C} \pm 2^{\circ} \mathrm{C}$, at relative humidity of $78 \pm 2 \%$, and photo phase of $12 \mathrm{~L}$ : $12 \mathrm{D}$ for their growth and development [50]. They were provided with Cerelac and fish food powders on the ratio of $2: 1$ on a daily basis [32]. Pupae were collected using droppers and transferred in emerging cages of $30 \times 30 \times 30 \mathrm{~cm}$ in round metal containers to prevent them from flying away when they emerge into adults. The emerging adult mosquitoes 
were supplied with cotton wool dipped in $10 \%$ glucose solution [48].

2.4. Susceptibility Test. Susceptibility bioassays were carried out using insecticide susceptibility kits [51, 52]. Mosquitoes were exposed to papers impregnated with the WHO-recommended discriminating concentrations $(v / w)$ of $(0.05 \%)$ deltamethrin and $(0.75 \%)$ permethrin [52]. Controls were exposed to clean paper impregnated with silicon oil. The female mosquitoes from larvae that were collected from the rice paddy fields 3-5 days after prominence were used for the bioassay test, and the susceptible Kisumu strain was used for reference. The knockdown $(\mathrm{KD})$ rates were recorded at 10 , $1520,30,40,50$, and 60 minutes after being exposed to the insecticides and when the KD was less than $80 \%$ was observed after 60 minutes [51, 53]. Mosquitoes were, then, transferred into the paper cups and fed with glucose solution. Mortality was recorded after a $24 \mathrm{~h}$ holding period; during this time, mosquitoes were provided with a $10 \%$ sugar solution $[54,55]$. In the experimental rooms, heaters were used to rise temperature when the room temperature dropped, and the floors were wetted to cool the temperature when it rises above the recommended temperature of $25 \pm 2^{\circ} \mathrm{C}[52]$.

2.5. Monitoring Longevity of An. gambiae s.l. Three-day-old female mosquitoes were exposed to deltamethrin and permethrin (the commonly used pyrethroid insecticide for public health) for an hour; the mosquitoes were, then, placed in a paper cup and were given $10 \%$ glucose solution. After 24 hours, the survived mosquitoes were transferred in a cage. They were provided with blood meal. The longevity of a mosquito was monitored until death to mimic the time after exposure of wild population when they contacted insecticide-treated surfaces. Monitoring was after every $24 \mathrm{hr}$. The dead mosquitoes were counted and removed daily from the cage [56].

2.6. Monitoring Feeding Succession of Anopheles gambiae s.l. The effect of the insecticide resistance on the feeding succession of An. gambiae s.l. was monitored through provision of a blood meal source and monitoring the number successfully fed. The tested vectors were offered blood meal twice a week; the first blood meal was given 48 hours after the susceptibility test was performed. The blood meal was always given in the morning times between 07:00 and 09: $00 \mathrm{~h}$ after being sugar-deprived for one hour before the blood meal [57]. Rabbits' fur was shaved dorsal ventrally, and then, they were kept in a restrainer, which limited their movement and exposed the shaven part to mosquitoes to feed for 30 minutes, but the number of successfully fed mosquitoes was recorded after one hour. In order to enhance the feeding process, the cages were covered with dark clothes [58]. Rabbits used to provide the blood meal were obtained from the TPRI laboratory facilities within the Mabogini paddy rice irrigation scheme. The feeding succession was

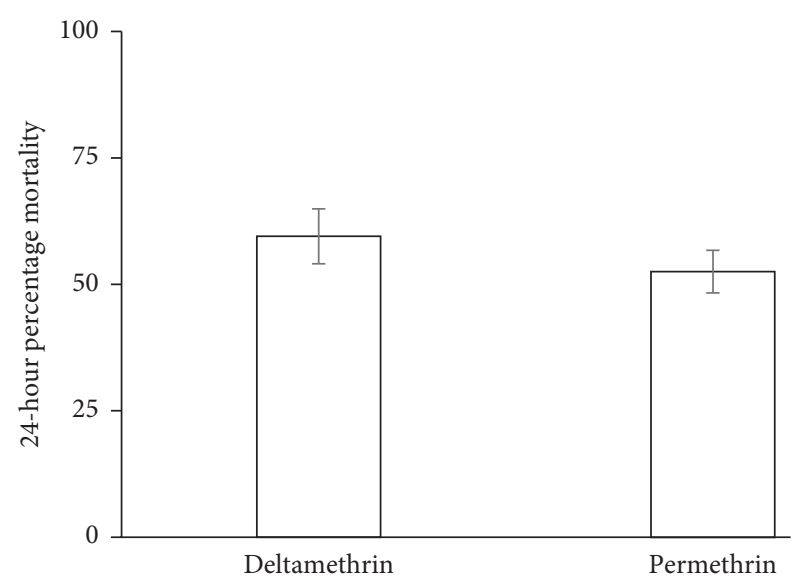

FIgURE 1: Mortality of wild Anopheles gambiae s.l. exposed to deltamethrin and permethrin treatments.

determined by counting the number of mosquitoes that successfully fed on blood during the 30 minutes.

2.7. Monitoring Egg Batch Size of An. gambiae s.l. After mosquitoes were fed on rabbits for a blood meal, they laid eggs on a wet white filter paper (Whatman No. 1, diameter $9 \mathrm{~cm}$ ) placed on a plastic transparent Petri dish. Collection of eggs was performed at an interval of three days after every blood meal, and then, the eggs were counted with the aid of a dissecting microscope $[59,60]$. After egg counting, the effect of insecticide tolerance on egg batch size of mosquitoes was assessed by comparing the mean number of eggs that were produced from a resistant colony against that of the susceptible colony.

2.8. Data Analysis. Data were analyzed using SAS software version 9.3 (SAS Institute Inc. 2008). The generalized linear model (GLM) was used to compare the mean difference between treatments, day and time, and their interactions. The parameters were tested separately for the three treatments (deltamethrin, permethrin, and control) at 95\% confidence level. The Bonferroni correction was used to adjust for multimeans comparisons. Fifty percent (50\%) and ninety-five percent (95\%) knockdown times $\left(\mathrm{KDT}_{50}\right.$ and $\mathrm{KDT}_{95}$ ) for both resistant and susceptible samples were estimated using the log-time probit model. The Kaplan-Meier analysis was used to estimate the longevity of mosquitoes. The comparison between the survivorship curves (susceptible and resistant) was performed using the Wilcoxon signed rank test.

\section{Results}

3.1. Insecticides Susceptibility Status. The susceptibility test was conducted using 3-5-day-old unfed female mosquitoes, and in this study, it was found that the resistance status of wild An. gambiae s.l. populations was based on decreased mortality rates to permethrin (mortality rate 52.5\%) and deltamethrin (mortality rate $=59.5 \%$ ). The mortality rate for the susceptible reference Kisumu strain was $100 \%$. Also, the 


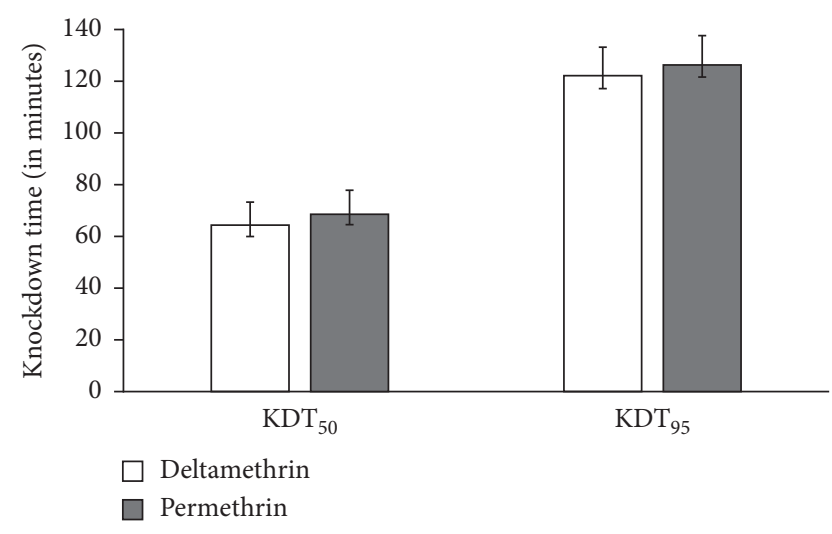

Figure 2: The mean $\mathrm{KDT}_{50}$ and $\mathrm{KDT}_{95}$ for deltamethrin and permethrin against Anopheles gambiae s.l.

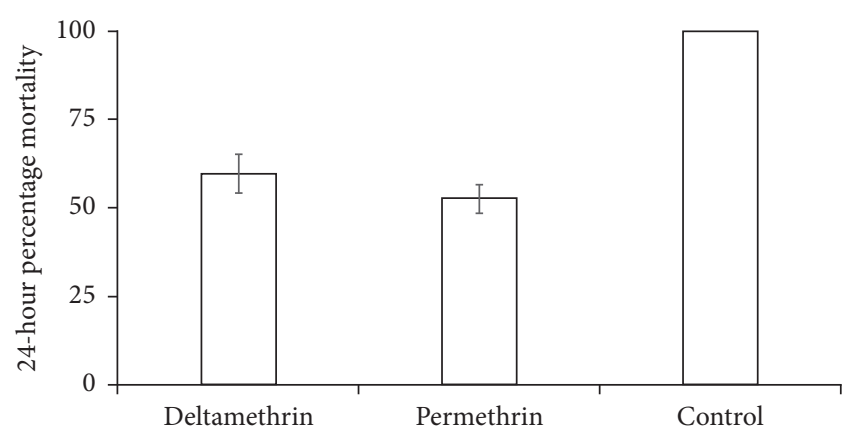

Figure 3: Mortality of wild Anopheles gambiae s.l. exposed to different treatments (i.e., deltamethrin, permethrin, and control) (single factor ANOVA, $P=0.001$ ).

study found out that resistance to pyrethroids was matched with increased knockdown times with the $\mathrm{KDT}_{95}<20$ mins for both pyrethroids. The knockdown time $\mathrm{KDT}_{50}$ varied from 64.52 to $68.83 \mathrm{~min}$ and $\mathrm{KDT}_{95}$ varied from 122.7 to 126. 48 mins for deltamethrin and permethrin, respectively. There was no significant difference, which was observed in the insecticides susceptibility status between deltamethrin and permethrin against An. gambiae s.l. $\left(F_{(1,15)}=1.294\right.$, $P=0.599$ ) (Figure 1). However, there was a significant difference in the knockdown time at $\mathrm{KDT}_{95}$ as indicated in Figure 2. There was no significant difference in mortality when the wild An. gambiae s.l. were treated with deltamethrin and permethrin insecticides, as shown in Figure 1 $\left(F_{(2,15)}=0.923, P=0.132\right)$, but mortality of the wild $A n$. gambiae s.l. differed significantly when the two insecticides were compared with control treatment (susceptible colony) $\left(F_{(2,15)}=4.113, P=0.001\right)$ (Figure 3).

3.2. Longevity of the Insecticide-Resistant Colony against the Susceptible Colony. Longevity was measured from the time when mosquitoes have been exposed to insecticides and survived and obtain blood meals until the time when they died. Adult female mosquitoes were provided with blood meals twice per week, and $10 \%$ glucose were provided in between. The findings revealed that the maximum longevity was 27 days after exposure for the resistant wild An. gambiae

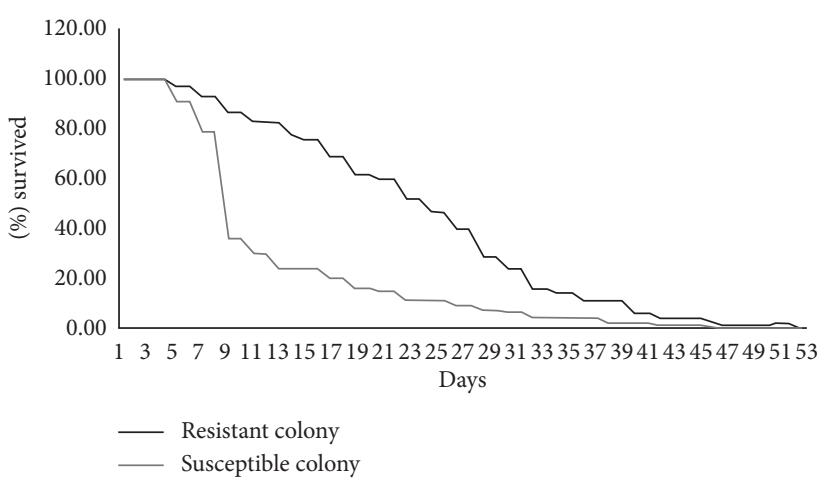

FIgURE 4: The proportion of resistance (An. gambiae s.l.) and susceptible (An. gambiae s.s.) survived after blood meal.

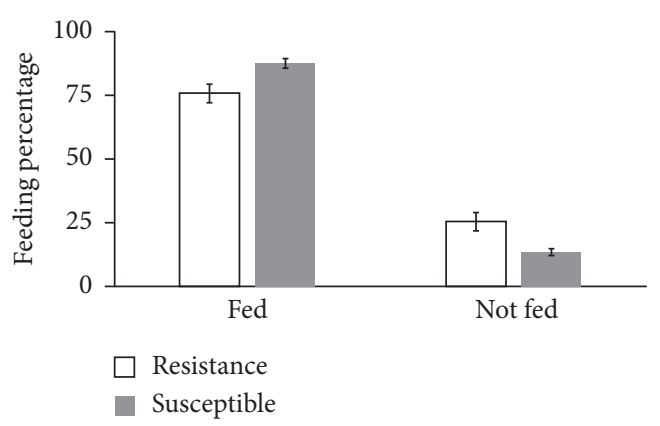

FIgUre 5: The feeding succession of Anopheles gambiae s.s. (Kisumu susceptible strain) and An. gambiae s.l. (wild resistant colonies).

s.l. and 24 days for the Kisumu susceptible strain. This indicated that the Kisumu susceptible colony lived for a shorter period of time than the wild female An. gambiae s.l., and its observed mean longevity was 26 and 20 days in control for the wild An. gambiae s.l. and the Kisumu susceptible strain, respectively. The Wilcoxon rank test was used to compare the resistant wild female An. gambiae s.l. and susceptible Kisumu strain $(Z=6.093, \mathrm{df}=86, P=0.001)$ (Figure 4).

\subsection{Feeding Succession of the Insecticide-Resistant Colony} against the Susceptible Colony. Blood feeding ability of wild female An. gambiae s.l after being exposed to the insecticides was tested for both strains (i.e., wild An. gambiae s.l and Kisumu susceptible strain). They were provided with blood twice per week, and the number of fed and unfed mosquitoes was jotted down. In wild strain, a total of 157 An. gambiae s.l that survived the exposure against the two pyrethroids tested were used. It was observed that $119(75.7 \%)$ mosquitoes fed successfully on blood and the remaining 38 (24.3\%) did not feed. Furthermore, a total of 400 Kisumu susceptible strain were also fed on blood, and 358 (89.6\%) successfully fed on blood, while the remaining 42 (10.4\%) did not feed. Statistical analysis indicates that the numbers of female mosquitos fed on blood meals were significantly higher in the Kisumu susceptible strain than in the wild resistant colony $(t=2.789, \mathrm{df}=21, P=0.011$ (Figure 5). 


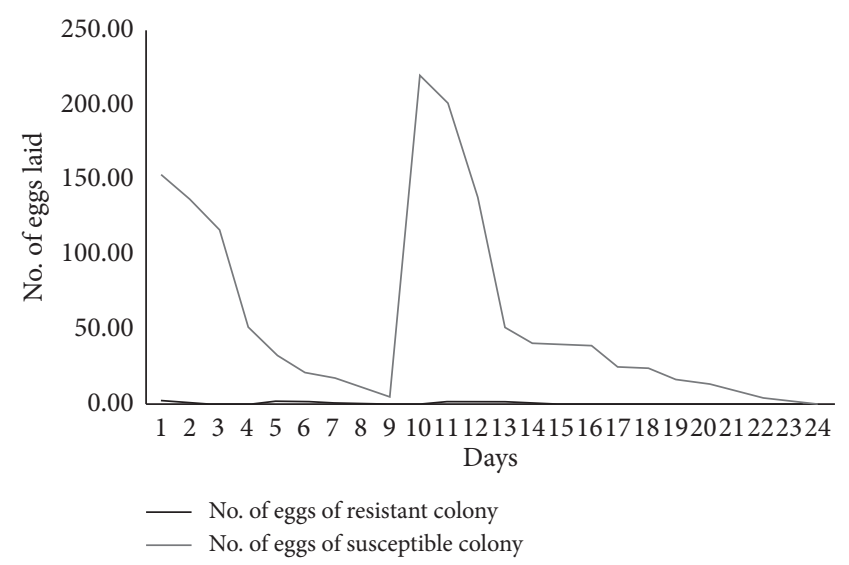

Figure 6: The egg batch sizes of Kisumu susceptible strain (An. gambiae s.s.) and (An. gambiae s.l.) wild resistant colonies.

3.4. Egg Batch Size of the Insecticide-Resistant Colony versus the Susceptible Colony. The ability of mosquitoes to lay viable eggs and develop into the next generation is another measure of mosquito success in malaria transmission. In this study, the number of eggs that were laid by both strains/ colonies was counted and their proportions were compared. The study revealed that the egg batch size of the wild $A n$. gambiae s.l. was affected by the two pyrethroids tested. A total of 1409 eggs were laid by both Kisumu susceptible strain and wild An. gambiae s.l., whereas 1392 out of these 1409 eggs were laid by the Kisumu susceptible strain and only 17 were laid by the wild An. gambiae s.l. that was considered as a resistant colony. Therefore, the study revealed the insecticide resistance impact on the egg-laying ability of the vector (An. gambiae s.l.). The Kisumu susceptible colony had laid more eggs than wild female $A n$. gambiae s.l., and the difference was significantly different by $X^{2}=1366, \mathrm{df}=1, P \leq 0.05$ (Figure 6).

\section{Discussion}

The findings of this study show that insecticide resistance has a great impact on susceptibility, egg batch size, longevity, and feeding success of malaria vectors, An. gambiae s.l.. The results in this study illustrated that the wild $A n$. arabiensis collected from irrigation schemes at Mabogini are resistant to the two pyrethroids (deltamethrin and permethrin). Their tolerance observed to pyrethroids could be elaborated by the fact that the vectors are repeatedly exposed to the pyrethroids in this study area [27, 32, 33, 45, 61, 62]. This is because, in the studied area, the main income-generating activity is agriculture, both animal keeping and crop production (rice), with intensive use of pyrethroid-based insecticides for veterinary, public health, and agricultural purposes, as reported by several studies conducted in the same vicinity [27, 32, 33, 45, 61, 62]. Therefore, mosquitoes might have developed resistance as a result of everyday contact with treated cattle during blood meal because the dominant species in the study area is An. arabiensis, and this species preferentially feeds on cattle blood as opposed to human blood [63-66]. Furthermore, vector resistance might have resulted as an effect of frequent exposure to insecticides residues in farms from the egg to pupa stages in the rice fields. These results are similar to previous studies that reported resistance in wild An. gambiae s.l. to pyrethroids, in lower Moshi, northeastern Tanzania, and in Muleba, northwestern Tanzania [30, 32, 33, 61].

In this study, the longevity of a vector was defined as the time mosquitoes survived the first 24 hours after exposure to deltamethrin/permethrin until actual death. Longevity is an important parameter when considering mosquito competence in transmitting malaria disease; the mosquito must survive long enough so that the Plasmodium life cycle will be complete and be transferred to the salivary gland of the vector [57]. The difference in longevity between wild mosquitoes and susceptible colonies showed that the resistant population had shown to survive longer than susceptible ones. These findings were supported with the former studies conducted in a laboratory in Switzerland where increase in longevity was observed in resistant colonies [67]. Similarly, in South Africa, Okoye and others reported longer life span among females of An. funestus compared to their susceptible counterparts [65]. However, findings of this study are in contrary to the previous two studies in which longevity was lower on resistant colonies [37]. An increase in the longevity that was observed in the resistant colony in this study could be a threat to the ongoing malaria control efforts and eradication agenda that were set by WHO for the purpose of eliminating malaria globally [67]. Although resistance management strategies often rely on the assumption of reduced fitness in vector populations associated with resistance genes, studies in other insects such as boll weevils, houseflies, and cockroaches do not show differences in fitness between resistant and susceptible strains [68, 69]. Nevertheless, there is no uniform negative effect of fitness costs associated with resistance across species, and the negative performance in one parameter can conceivably be balanced by the positive performance in another [70]. In the wild, various environmental factors including food and temperature affect developmental rates and survivorship of mosquito immature and, subsequently, the adult life span.

The feeding succession is an important parameter of mosquitoes in transmitting disease; therefore, inability to feed on blood can change the transmission dynamics which, in turn, will severely limit their ability to transmit disease [67]. The numbers of fed and unfed mosquitoes were counted after each blood meal for both strains [57]. From the findings of this study, it can be seen that the feeding succession of a wild An. gambiae s.l. was reduced and the percentage of unfed An. gambiae s.l. was higher in the wild An. gambiae s.l. compared to the susceptible colony. This shows that the resistant population of An. gambiae s.l. can be denied an opportunity to feed when the host is well protected with LLINs [67]. The results of this study on feeding succession were corroborated by other studies conducted elsewhere [36, 37].

In this study, the resistance character of a vector negatively affected the number of eggs. The wild female $A n$. gambiae s.l. laid less number of eggs compared to those which were laid by the susceptible strain. According to 
Couret and others in their studies, they interpreted a model that illustrated the interaction between larval population size and adult population size and how this model was affected by the number of eggs laid by a mosquito [71]. Smaller number of eggs that would be produced and laid by the resistant colony would have an impact on the number of larvae produced and adult populace that will exist. The number of eggs that were produced by the resistant strain was less compared to that produced by susceptible colony. Similar findings have been reported where resistant colonies showed reduction in egg batch sizes [72], and this information is of public health importance as it translates to reduced vector density.

\section{Conclusions}

The findings of this study have shown the high degree of tolerance to permethrin and deltamethrin insecticides among a wild population of An. gambiae s.l. Also, the findings showed an increased longevity of the resistant colony, decreased feeding habit, and decreased egg batch size of the resistant vectors compared to the susceptible colony. These results give an insight on the effects of insecticides resistant on malaria vector species and their impact to the spread of the malaria considering the greater extent nature of pyrethroids resistance in sub-Saharan Africa.

\section{Abbreviations}

LLIN: Long-lasting insecticidal nets

IRS: Indoor residual spray

WHO: World Health Organization

TPRI: Tropical Pesticides Research Institute

KDT: Knockdown time

KD: Knockdown.

\section{Data Availability}

Comment: Data can be availed upon request from the corresponding author.

\section{Conflicts of Interest}

The authors declare that they have no conflicts of interest.

\section{Authors' Contributions}

GM and EJK conceived the study, planned, and led the writing of this article. GM and AMM participated in data collection and analysis. AP and MO participated in writing the manuscript and reviewed continuously. All authors read, revised, and approved the final version of the manuscript.

\section{Acknowledgments}

Authors are thankful to the TPRI staff at Mabogini field station who, in many ways, participated in data collection activities. The TPRI is highly appreciated for providing its research infrastructure for the completion of this research.

\section{References}

[1] WHO, World Malaria Report 2019, World Health Organisation, Geneva, Switzerland, 9241565659, 2019.

[2] A. Asale, D. Kussa, M. Girma, C. Mbogo, and C. M. Mutero, "Community based integrated vector management for malaria control: lessons from three years' experience (2016-2018) in Botor-Tolay district, southwestern Ethiopia," BMC Public Health, vol. 19, no. 1, p. 1318, 2019.

[3] S. Kibret, G. G. Wilson, D. Ryder, H. Tekie, and B. Petros, "The influence of dams on malaria transmission in sub-Saharan Africa," EcoHealth, vol. 14, no. 2, pp. 408-419, 2017.

[4] E. O. Ogola, E. Chepkorir, R. Sang, and D. P. Tchouassi, “A previously unreported potential malaria vector in a dry ecology of Kenya," Parasites \& Vectors, vol. 12, no. 1, p. 80, 2019.

[5] E. O. Ogola, U. Fillinger, and I. M. Ondiba, "Insights into malaria transmission among Anopheles funestus mosquitoes, Kenya," Parasites \& Vectors, vol. 11, no. 1, p. 577, 2018.

[6] E. K. Grover-Kopec, M. B. Blumenthal, P. Ceccato, T. Dinku, J. A. Omumbo, and S. J. Connor, "Web-based climate information resources for malaria control in Africa," Malaria Journal, vol. 5, no. 1, p. 38, 2006.

[7] E. Grover-Kopec, M. Kawano, R. W. Klaver, B. Blumenthal, P. Ceccato, and S. J. Connor, "An online operational rainfallmonitoring resource for epidemic malaria early warning systems in Africa," Malaria Journal, vol. 4, no. 1, p. 6, 2005.

[8] M. E. Sinka, M. J. Bangs, S. Manguin et al., "A global map of dominant malaria vectors," Parasites \& Vectors, vol. 5, no. 1, p. 69, 2012.

[9] E. J. Kweka, H. D. Mazigo, L. J. Lyaruu et al., “Anopheline mosquito species composition, kdr mutation frequency, and parasite infectivity status in northern Tanzania," Journal of Medical Entomology, vol. 57, no. 3, pp. 933-938, 2020.

[10] A. Burke, L. Dandalo, G. Munhenga et al., "A new malaria vector mosquito in South Africa," Scientific Reports, vol. 7, no. 1, Article ID 43779, 2017.

[11] E. J. Kweka, A. M. Mahande, W. M. Nkya et al., "Vector species composition and malaria infectivity rates in Mkuzi," Muheza District, North-Eastern Tanzania, vol. 10, no. 1, pp. 46-49, 2008.

[12] A. Burke, Y. Dahan-Moss, F. Duncan et al., "Anopheles parensis contributes to residual malaria transmission in South Africa," Malaria Journal, vol. 18, no. 1, p. 257, 2019.

[13] E. J. Kweka, L. Kamau, S. Munga, M.-C. Lee, A. K. Githeko, and G. Yan, "A first report of Anopheles funestus sibling species in western Kenya highlands," Acta Tropica, vol. 128, no. 1, pp. 158-161, 2013.

[14] E. J. Kweka, G. Zhou, M.-C. Lee et al., "Evaluation of two methods of estimating larval habitat productivity in western Kenya highlands," Parasites \& Vectors, vol. 4, no. 1, p. 110, 2011.

[15] E. J. Kweka, W. M. M. Nkya, A. M. Mahande et al., "Mosquito abundance, bed net coverage and other factors associated with variations in sporozoite infectivity rates in four villages of rural Tanzania," Malaria Journal, vol. 7, no. 1, p. 59, 2008.

[16] H.-M. Liu, P.-P. Yang, P. Cheng et al., "Resistance level of mosquito species (Diptera: Culicidae) from shandong province, China," International Journal of Insect Science, vol. 7, Article ID S24232, 2015.

[17] T. R. Burkot, R. Farlow, M. Min, E. Espino, A. Mnzava, and T. L. Russell, "A global analysis of National Malaria Control Programme vector surveillance by elimination and control status in 2018," Malaria Journal, vol. 18, no. 1, p. 399, 2019. 
[18] S. Wanji, T. M. Nji, L. Hamill et al., "Implementation of testand-treat with doxycycline and temephos ground larviciding as alternative strategies for accelerating onchocerciasis elimination in an area of loiasis co-endemicity: the COUNTDOWN consortium multi-disciplinary study protocol," Parasites \& Vectors, vol. 12, no. 1, p. 574, 2019.

[19] A. K. Musiime, D. L. Smith, M. Kilama et al., "Impact of vector control interventions on malaria transmission intensity, outdoor vector biting rates and Anopheles mosquito species composition in Tororo, Uganda," Malaria Journal, vol. 18, no. 1, p. 445, 2019.

[20] WHO, World Malaria Report 2018, World Health Organisation, Geneva, Switzerland, 9241565659, 2018.

[21] T. Gari and B. Lindtjørn, "Reshaping the vector control strategy for malaria elimination in Ethiopia in the context of current evidence and new tools: opportunities and challenges," Malaria Journal, vol. 17, no. 1, p. 454, 2018.

[22] O. Kenea, M. Balkew, H. Tekie et al., "Human-biting activities of Anopheles species in south-central Ethiopia," Parasites \& Vectors, vol. 9, no. 1, p. 527, 2016.

[23] O. Kenea, M. Balkew, H. Tekie et al., "Impact of combining indoor residual spraying and long-lasting insecticidal nets on Anopheles arabiensis in Ethiopia: results from a cluster randomized controlled trial," Malaria Journal, vol. 18, no. 1, p. 182, 2019.

[24] W. N. Kisinza, T. E. Nkya, B. Kabula et al., "Multiple insecticide resistance in Anopheles gambiae from Tanzania: a major concern for malaria vector control," Malaria Journal, vol. 16, no. 1, p. 439, 2017.

[25] A. Rivero, J. Vézilier, M. Weill, A. F. Read, and S. Gandon, "Insecticide control of vector-borne diseases: when is insecticide resistance a problem?" PLoS Pathogens, vol. 6, no. 8, Article ID e1001000, 2010.

[26] A. Philbert, S. L. Lyantagaye, and G. Nkwengulila, "Farmers' pesticide usage practices in the malaria endemic region of North-Western Tanzania: implications to the control of malaria vectors," BMC Public Health, vol. 19, no. 1, p. 1456, 2019.

[27] D. Yewhalaw and E. J. Kweka, "Insecticide resistance in East Africa-history, distribution and drawbacks on malaria vectors and disease control," in Insecticides Resistance, S. Trdan, Ed., 39 Intech, Rijeka, Croatia, pp. 189-215, 2016.

[28] W. G. Brogdon and J. C. McAllister, "Simplification of adult mosquito bioassays through use of time-mortality determinations in glass bottles," Journal of the American Mosquito Control Association, vol. 14, no. 2, pp. 159-164, 1998.

[29] N. Pasteur and M. Raymond, "Insecticide resistance genes in mosquitoes: their mutations, migration, and selection in field populations," Journal of Heredity, vol. 87, no. 6, pp. 444-449, 1996.

[30] J. Matowo, M. A. Kulkarni, F. W. Mosha et al., "Biochemical basis of permethrin resistance in Anopheles arabiensis from Lower Moshi, north-eastern Tanzania," Malaria Journal, vol. 9, no. 1, p. 193, 2010.

[31] M. Sarwar and M. Salman, "Insecticides resistance in insect pests or vectors and development of noveltrategies to combat its evolution," International Journal of Bioinformatics Biomedical Engineering, vol. 1, no. 3, pp. 344-351, 2015.

[32] S. Mbepera, G. Nkwengulila, R. Peter et al., "The influence of age on insecticide susceptibility of Anopheles arabiensis during dry and rainy seasons in rice irrigation schemes of Northern Tanzania," Malaria Journal, vol. 16, no. 1, p. 364, 2017.
[33] E. J. Nnko, C. Kihamia, F. Tenu, Z. Premji, and E. J. Kweka, "Insecticide use pattern and phenotypic susceptibility of Anopheles gambiae sensu lato to commonly used insecticides in Lower Moshi, northern Tanzania," BMC Research Notes, vol. 10, no. 1, p. 443, 2017.

[34] P. Khemrattrakool, J. Yanola, N. Lumjuan, and P. Somboon, "Pyriproxyfen-treated polypropylene sheets and resting boxes for controlling mosquitoes in livestock operations," Insects, vol. 10, no. 2, p. 55, 2019.

[35] S. Kumar, A. Molina-Cruz, L. Gupta, J. Rodrigues, and C. Barillas-Mury, "A peroxidase/dual oxidase system modulates midgut epithelial immunity in Anopheles gambiae," Science, vol. 327, no. 5973, pp. 1644-1648, 2010.

[36] S. Kumar, A. Thomas, T. Samuel, A. Sahgal, A. Verma, and M. J. T. B. Pillai, "Diminished reproductive fitness associated with the deltamethrin resistance in an Indian strain of dengue vector mosquito, 4 Aedes aegypti L." Tropical Biomedicine, vol. 26, no. 2, pp. 155-164, 2009.

[37] F. W. Mosha, I. N. Lyimo, R. M. Oxborough et al., "Comparative efficacies of permethrin-, deltamethrin- and $\alpha$-cypermethrin-treated nets, against Anopheles arabiensis and Culex quinquefasciatus in northern Tanzania," Annals of Tropical Medicine \& Parasitology, vol. 102, no. 4, pp. 367-376, 2008.

[38] J. M. Darbro, S. A. Ritchie, M. B. Thomas, P. H. Johnson, P. A. Ryan, and B. H. Kay, "Effects of beauveria bassiana on survival, blood-feeding success, and fecundity of Aedes aegypti in laboratory and semi-field conditions," The American Journal of Tropical Medicine and Hygiene, vol. 86, no. 4, pp. 656-664, 2012.

[39] S. V. Oliver and B. D. Brooke, "The effect of multiple bloodfeeding on the longevity and insecticide resistant phenotype in the major malaria vector Anopheles arabiensis (Diptera: Culicidae)," Parasites \& Vectors, vol. 7, no. 1, p. 390, 2014.

[40] World Health Organization, Instructions for Determining the Susceptibility or Resistance of Mosquito Larvae to Insecticides Unpublished Document WHO/VBC/81, World Health Organization, Geneva, Switzerland, 1981.

[41] A. Cohuet, C. Harris, V. Robert, and D. Fontenille, "Evolutionary forces on Anopheles: what makes a malaria vector?" Trends in Parasitology, vol. 26, no. 3, pp. 130-136, 2010.

[42] H. Alout, B. Roche, R. K. Dabiré, and A. Cohuet, "Consequences of insecticide resistance on malaria transmission," PLoS Pathogens, vol. 13, no. 9, Article ID e1006499, 2017.

[43] M. Raymond, C. Berticat, M. Weill, N. Pasteur, and C. Chevillon, "Insecticide resistance in the mosquito Culex pipiens: what have we learned about adaptation?" Microevolution Rate, Pattern, Process, Contemporary Issues in Genetics and Evolution, vol. 112-113, no. 1, pp. 287-296, 2001.

[44] M. Viana, A. Hughes, J. Matthiopoulos, H. Ranson, and H. M. Ferguson, "Delayed mortality effects cut the malaria transmission potential of insecticide-resistant mosquitoes," Proceedings of the National Academy of Sciences, vol. 113, no. 32, pp. 8975-8980, 2016.

[45] A. M. Mahande, I. Dusfour, J. R. Matias, and E. J. Kweka, "Knockdown resistance, Rdl alleles, and the annual entomological Inoculation rate of wild mosquito populations from Lower Moshi, Northern Tanzania," Journal of Global Infectious Diseases, vol. 4, no. 2, pp. 114-119, 2012.

[46] J. N. Ijumba, F. C. Shenton, S. E. Clarke, F. W. Mosha, and S. W. Lindsay, "Irrigated crop production is associated with less malaria than traditional agricultural practices in Tanzania," Transactions of The Royal Society of Tropical Medicine and Hygiene, vol. 96, no. 5, pp. 476-480, 2002. 
[47] E. J. Kweka, F. Mosha, and A. Lowassa, "Ethnobotanical study of some of mosquito repellent plants in north-eastern Tanzania," Malaria Journal, vol. 7, no. 1, p. 152, 2008.

[48] WHO, Manual On Practical Entomology. Part II. Methods And Techniques, World Health Organization, Geneva, Switzerland, 1975.

[49] M. T. Gillies and B. De Meillon, "The Anophelinae of Africa south of the Sahara (Ethiopian zoogeographical region)," Mosquito Taxonomic Inventory, vol. 54, pp. 1-343, 1968.

[50] H. S. Kivuyo, P. H. Mbazi, D. S. Kisika et al., "Performance of five food regimes on Anopheles gambe senso stricto larval rearing to adult emergence in insectary," PLoS One, vol. 9, no. 10, Article ID e110671, 2014.

[51] WHO, Guidelines for Laboratory and Field-Testing of LongLasting Insecticidal Nets, World Health Organization, Geneva, Switzerland, 9241505273, 2013.

[52] WHO, Test Procedures for Insecticide Resistance Monitoring in Malaria Vectors, Bio-Efficacy and Persistence of Insecticides on Treated Surfaces: Report of the WHO Informal Consultation, World Health Organization, Geneva, Switzerland, 1998.

[53] WHO, Test Procedures for Insecticides Resistance Monitoring in Malaria Vector Mosquitoes, World Health Organisation, Geneva, Switzerland, 2016.

[54] J. Matowo, J. Kitau, R. Kaaya et al., "Trends in the selection of insecticide resistance inAnopheles gambiae s.l.mosquitoes in northwest Tanzania during a community randomized trial of longlasting insecticidal nets and indoor residual spraying," Medical and Veterinary Entomology, vol. 29, no. 1, pp. 51-59, 2015.

[55] E. Ochomo, M. N. Bayoh, W. G. Brogdon et al., "Pyrethroid resistance in Anopheles gambiae s.s. and Anopheles arabiensis in western Kenya: phenotypic, metabolic and target site characterizations of three populations," Medical and Veterinary Entomology, vol. 27, no. 2, pp. 156-164, 2013.

[56] WHO, Manual on Practical Entomology in Malaria. Part II. Methods and Techniques, World Health Organization, Geneva, Switzerland, 1975.

[57] M. Mulatier, A. Porciani, L. Nadalin et al., "DEET efficacy increases with age in the vector mosquitoes Anopheles gambiae s.s. and Aedes albopictus (Diptera: Culicidae)," Journal of Medical Entomology, vol. 55, no. 6, pp. 1542-1548, 2018.

[58] D. Damiens, S. M. Soliban, F. Balestrino, R. Alsir, M. J. B. Vreysen, and J. R. L. Gilles, "Different blood and sugar feeding regimes affect the productivity of Anopheles arabiensis colonies (Diptera: Culicidae)," Journal of Medical Entomology, vol. 50, no. 2, pp. 336-343, 2013.

[59] L. A. Sumba, K. Okoth, A. L. Deng et al., "Daily oviposition patterns of the African malaria mosquito Anopheles gambiae Giles (Diptera: Culicidae) on different types of aqueous substrates," Journal of Circadian Rhythms, vol. 2, no. 1, p. 6, 2004.

[60] W. Mamai, N. S. Bimbile-Somda, H. Maiga et al., "Optimization of mosquito egg production under mass rearing setting: effects of cage volume, blood meal source and adult population density for the malaria vector, Anopheles arabiensis," Malaria Journal, vol. 16, no. 1, p. 41, 2017.

[61] J. Matowo, C. M. Jones, B. Kabula et al., "Genetic basis of pyrethroid resistance in a population of Anopheles arabiensis, the primary malaria vector in Lower Moshi, north-eastern Tanzania," Parasites \& Vectors, vol. 7, no. 1, p. 274, 2014.

[62] C. L. Wanjala and E. J. Kweka, "Malaria vectors insecticides resistance in different agroecosystems in western Kenya," Front Public Health, vol. 6, no. 55, 2018.
[63] A. K. Githeko, M. W. Service, C. M. Mbogo, F. K. Atieli, and F. O. Juma, "Origin of blood meals in indoor and outdoor resting malaria vectors in western Kenya," Acta Tropica, vol. 58, no. 3-4, pp. 307-316, 1994.

[64] A. M. Mahande, F. W. Mosha, J. M. Mahande, and E. J. Kweka, "Role of cattle treated with deltamethrine in areas with a high population of Anopheles arabiensis in Moshi, Northern Tanzania," Malaria Journal, vol. 6, no. 1, p. 109, 2007.

[65] A. Mahande, F. Mosha, J. Mahande, and E. Kweka, "Feeding and resting behaviour of malaria vector, Anopheles arabiensis with reference to zooprophylaxis," Malaria Journal, vol. 6, no. 1, p. 100, 2007.

[66] C. M. Fornadel, D. E. Norris, G. E. Glass, and L. C. Norris, "Analysis of Anopheles arabiensis blood feeding behavior in southern Zambia during the two years after introduction of insecticide-treated bed nets," The American Journal of Tropical Medicine and Hygiene, vol. 83, no. 4, pp. 848-853, 2010.

[67] A. M. G. Barreaux, C. M. Stone, P. Barreaux, and J. C. Koella, "The relationship between size and longevity of the malaria vector Anopheles gambiae (s.s.) depends on the larval environment," Parasites \& Vectors, vol. 11, no. 1, p. 485, 2018.

[68] R. T. Roush and M. A. Hoy, "Laboratory, glasshouse, and field studies of artificially selected carbaryl resistance in metaseiulus occidentalis 12," Journal of Economic Entomology, vol. 74, no. 2, pp. 142-147, 1981.

[69] J. G. Thomas and J. R. Brazzel, "A compartive study ofcertain biological phenomena of a resistant and a susceptible strain of the boll weevil, Anthonomus grandis," Journal of Economic Entomology, vol. 54, no. 3, pp. 417-420, 1961.

[70] P. N. Okoye, B. D. Brooke, R. H. Hunt, and M. Coetzee, "Relative developmental and reproductive fitness associated with pyrethroid resistance in the major southern African malaria vector, Anopheles funestus," Bulletin of Entomological Research, vol. 97, no. 6, pp. 599-605, 2007.

[71] J. Couret, E. Dotson, and M. Q. Benedict, “Temperature, larval diet, and density effects on development rate and survival of Aedes aegypti (Diptera: Culicidae)," PLoS One, vol. 9, no. 2, Article ID e87468, 2014.

[72] A. Djènontin, L. P. Ahoua Alou, A. Koffi et al., "Insecticidal and sterilizing effect of Olyset Duo, a permethrin and pyriproxyfen mixture net against pyrethroid-susceptible and -resistant strains ofAnopheles gambiae s.s.: a release-recapture assay in experimental huts," Parasite, vol. 22, p. 27, 2015. 